\title{
Teatralización en grupos tutorizados de Biología en Medicina: una estrategia diferente para el aprendizaje colaborativo.
}

\section{Teresa San-Miguel Díez ${ }^{a}$, Javier Megías Vericat ${ }^{b}$ y Eva Serna García}

${ }^{a}$ Departamento de Patología, Universitat de València. Teresa.miguel@uv.es bepartamento de Patología, Universitat de València. Javier.megias@uv.es y ${ }^{\mathrm{c}}$ Departamento de Fisiología, Universitat de València. Eva.serna@uv.es.

\begin{abstract}
Dramatization as a teaching methodology remains relatively unknown in higher education. It can be especially useful to work cross-disciplinary skills because of the cooperative nature that is intrinsic to the theater itself. The subjects and disciplines that can benefit from it must be adequately valued by teachers. Here we present an activity in small groups, focused on rare diseases with a known genetic cause, in which the theater is used as a motivating axis to gather information and organize it by creating a storyline.
\end{abstract}

Keywords: theater, TIE (theater in education), intrinsec motivation, rare diseases.

\begin{abstract}
Resumen
La teatralización como metodología de enseñanza es una gran desconocida en la educación superior. Su utilización para trabajar competencias transversales puede resultar especialmente útil por el carácter cooperativo intrínseco al propio teatro y las temáticas y disciplinas que pueden beneficiarse de ella deben ser adecuadamente valoradas por los docentes. Presentamos una actividad en grupos tutorizados, centrada en enfermedades raras con causa genética conocida, en la que el teatro sirve como eje motivador para recabar la información y organizarla creando un hilo argumental.
\end{abstract}

Palabras clave: teatro, MABAE (metodología de aprendizaje basado en artes escénicas), motivación intrínseca, enfermedades raras

\section{Introducción}

Durante la última década, los intentos de innovación en educación han sido una apuesta continua desde todas las administraciones. Con una aplicación más sencilla y temprana en las aulas de niveles preuniversitarios, es relativamente reciente el interés en la Universidad por aprender a enseñar (Durán, 2014), incentivar la actividad docente y formar mejor al profesorado dentro del Espacio Europeo de Educación Superior (EEES). En este sentido, la Universitat de València convoca proyectos de Innovación Educativa de manera periódica, y se están aumentando los recursos con, por ejemplo, programas de formación del profesorado como son el Pla de Formació d'Impuls al Lideratge Docent (PILD) o la Formación Integral para el Profesorado Universitario (FIPU). 
Así, se han puesto en marcha diferentes fórmulas para favorecer el desarrollo de procesos educativos innovadores (Peña, 2014), y escuchamos de manera cotidiana, nombrar metodologías como el "aprendizaje cooperativo o colaborativo", "aprendizaje basado en proyectos”, “aprendizaje basado en problemas”, "puzles de Aaronson”, "blended-learning” o "gamificación". Por otra parte, se nos explica la necesidad de orientar nuestros mecanismos de evaluación a la adquisición de competencias y no meramente a los contenidos; en este ámbito también aparecen nomenclaturas propias para los nuevos sistemas de evaluación que se nos sugieren, como evaluación por pares, evaluación entre iguales, evaluación combinada, sumativa, o evaluación mediante rúbricas entre muchas otras.

Ante tal variedad terminológica y diversidad de enfoque, el problema es que la mayoría continua enseñando y aprendiendo con idénticas estrategias que hace décadas (Acaso, 2013). Al profesor se le exige la actualización en el formato pedagógico mientras que el alumno permanece expectante (Álvarez-Domínguez, 2016) y la implementación del cambio es compleja. No es descabellado tratar de buscar herramientas pedagógicas integrativas que nos permitan, por una parte innovar en la metodología y por supuesto, lograr nuestro objetivo didáctico a la par que los estudiantes adquieren un papel más protagonista en las competencias que pretendemos que alcancen. En este sentido, las artes escénicas como metodología de enseñanza-aprendizaje en la universidad, resultan un campo inexplorado y con un gran potencial en cualquier disciplina. Evidentemente, hay grados en los que será más utilizable en primeros cursos y otros en los que se puede aplicar de manera puntual, en cualquier momento del proceso formativo de los estudiantes.

Ante la necesidad de bautizar todo método de enseñanza-aprendizaje que se introduce en el aula, estaríamos hablando de la ·Metodología de Aprendizaje Basado en las Artes Escénicas (MABAE), o más simplificado en inglés Theater in education (TIE). La teatralización puede lograr un aprendizaje holístico, más transversal y que conecta contenidos y emociones. Los estudiantes pueden integrar el aprendizaje basado en problemas o en proyectos y el apredizaje cooperativo, siendo ellos auténticamente los protagonistas de sus aprendizajes. La técnica se conoce como "Role-playing” y es sin duda, novedosa como concepto pedagógico dentro del aula universitaria (Navarro, 2010). Además, se trata de una técnica que potencia la motivación intrínseca del alumnado: el hecho de tener que teatralizar crea en el alumno-actor la necesidad de conocer su papel y por tanto, de comprender mejor los aspectos que va a representar.

En el caso concreto de este trabajo, se escogió el método para lograr el trabajo colaborativo que se espera cuando los alumnos realizan trabajos en grupo. Tradicionalmente consistía una exposición oral, por lo que los alumnos, que ya trabajan con herramientas on-line como Google Drive para, de acuerdo al sistema blended-learning, poder trabajar tanto presencialmente como de manera virtual, tendían a repartirse las tareas de manera completamente fragmentada, cayendo en lo que se conoce como balcanismo del conocimiento, y memorizando lo que los otros compañeros han aportado a cada una de sus separadas partes del trabajo. Al introducir la teatralización, los papeles de los personajes que participan interactúan entre ellos y tienen, en muchos casos, conocimientos solapantes,

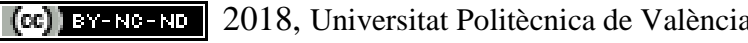


de manera que durante la búsqueda de información, que sí, sigue siendo fragmentada, deben compartir sus hallazgos, ponerlos en común y consensuar el mensaje final para evitar contradicciones y lograr una línea argumental coherente. El teatro actúa como vehículo para que el alumno tome conciencia de su propio aprendizaje y lo encarne. El hecho de teatralizar la temática, nos permite incluir (Torre, 2005):

- Concienciación de la temática

- Sentimiento y experimentación de la información como un contenido que afecta al propio individuo

- Reflexión sobre el tema en términos de acción

- Ejecución de la práctica a la propuesta escénica

- Integración y trascendencia de lo expuesto

Aprender de manera diferente es un reto en Educación Superior y, por ello, el papel del profesor como facilitador y guía es imprescindible para estructurar adecuadamente los planteamientos metodológicos que se quieren introducir. En base a esto, desarrollamos la actividad que a continuación se describe.

\section{Objetivos}

El objetivo principal es que los alumnos conozcan el concepto general de "enfermedades raras” y comprendan en profundidad una de ellas, de origen genético conocido, con sus repercusiones clínicas (la enfermedad a nivel genético, organular, celular y tisular), familiares (tratamiento, estudio genético y recomendaciones) y sociales (investigación, asociaciones, perspectivas).

La temática del trabajo pretende que los alumnos realicen un trabajo de investigación en grupo para trabajar en las competencias específicas del grado y en las de la asignatura de Biología: conocer la estructura y función celular y la implicación de las biomoléculas, conocer los mecanismos de de la información, expresión, regulación génica así como los patrones de herencia, comprender las consecuencias de una alteración genética puntual y su relación con fenotipos complejos, saber utilizar las tecnologías de la información y comunicación en actividades clínicas, terapéuticas, preventivas y de investigación.

Además, se pretenden desarrollar las competencias transversales propias de un profesional de la Medicina, y para ello se diseña la actividad como un proyecto teatralizado. Las competencias transversales a trabajar son:

CT1: Organizar y planificar adecuadamente la carga de trabajo

CT2: Capacidad para trabajar en equipo y para relacionarse con otras personas del mismo o distinto ámbito profesional

CT3: Capacidad crítica y autocrítica

CT4: Capacidad para comunicarse con colectivos profesionales de otras áreas

(c)) EY-NC-ND 2018, Universitat Politècnica de València

Congreso In-Red (2018) 
CT5: Reconocimiento de la diversidad y multiculturalidad

CT6: Considerar la ética como valor primordial en la práctica profesional

\section{Desarrollo de la innovación}

\subsection{Destinatarios y planificación}

La actividad se desarrolló dentro del programa de grupos tutelados de primer curso del grado de Medicina. Los destinatarios fueron los alumnos matriculados en el grupo de prácticas L5 de la asignatura Biología (34446) de la Facultat de Medicina i Odontologia de la Universitat de València en el primer cuatrimestre del curso 2017-2018, lo que supone un total de 38 alumnos. Se realizó una sesión de presentación de la actividad el 23/10/17 en la que se explicó a los participantes la finalidad del trabajo y se proporcionaron las pautas para su ejecución así como los plazos y mecanismos de evaluación.

En primer lugar, el docente estructuró los grupos de trabajo y los comunicó en dicha sesión: se formaron un total de 5 grupos de 7-8 participantes cada uno. A continuación se explicó la temática: realizar un trabajo de investigación en grupo sobre un enfermedad de origen genético con afectación organular concreta. Los temas ofertados, a elegir por los grupos de trabajo fueron: Síndrome de la triple A y síndrome de Rett (afectación del núcleo celular), Síndrome de Hurler-Scheie, síndrome de Gaucher y síndrome de Hunter (afectación del lisosoma), Síndrome de Zellweger (afectación del peroxisoma), Distrofia muscular de Duchenne (afectación de la membrana plasmática), Neuropatía óptica de Lebber (afectación de la mitocondria). El contenido que debían abordar fue descrito en la misma sesión y compartido con los alumnos para que lo tuvieran por escrito. Debía comprender

- Aspectos genéticos: causa responsable de la alteración fenotípica

- Biología celular: descripción del orgánulo o esctructura celular implicada y alteraciones que sufre

- Fisiopatología de la enfermedad: cuadro clínico

- Otros: diagnóstico, pronóstico, terapia, investigaciones actuales y perspectivas futuras

- Conclusiones y bibliografía.

Se explicaron los documentos y actividades que debían ejecutarse para la evaluación de la actividad:

- Documento resumen: máximo 6 hojas

- $\quad$ Póster en formato congreso: explicación de formato.

- Presentación en formato ppt o similar: guía de la representación y decorados.

Además se especificó la fecha final para la presentación del documento resumen y la representación teatralizada, con una extensión máxima de 15 minutos y un turno de preguntas por parte del profesorado presente y los compañeros a todos los miembros del grupo sobre cualquiera de los aspectos tratados.

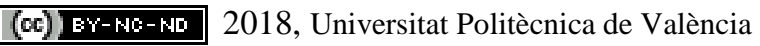

Congreso IN-RED (2018) 


\subsection{Estructura de la acción}

Se presentó el reparto de tareas para la teatralización, de manera que cada uno de los miembros del grupo debía adoptar uno de los roles planteados, con flexibilidad para su creatividad y adecuado a la patología (Fig. 1). De manera general en todos los grupos debía haber:

- Paciente: descripción de los síntomas como afectado, con terminología corriente.

- Médico: aporta la nomenclatura clínica de los síntomas y la sospecha diagnóstica como consecuencia

- Bioquímico-Genetista: realiza las pruebas diagnósticas solicitadas por el médico y aporta los resultados bioquímicos y genéticos correspondientes (gen, tipo de alteración, loci implicados cuando se identifique...)

- Profesor de Biología: caracteriza el orgánulo afectado en condiciones normales y los defectos consecuencia de la alteración y los relaciona con la fisiopatología

- Farmacéutico: describe el tratamiento sintomático, paliativo, dietético o experimental

- $\quad$ Padre/madre/hermano: averigua la epidemiología de la enfermedad, se implica en el tipo de herencia de cara a obtener un consejo genético, busca asociaciones de apoyo en España y perspectivas futuras para su familiar y para futuros afectados.

- Expertos polivalentes: para no obligar a la presentación teatralizada a los alumnos más tímidos, se ofrece la posibilidade destinar a 1-2 miembros a centrarse en la organización de los contenidos, desarrollo del guión y formatos escritos a presentar.

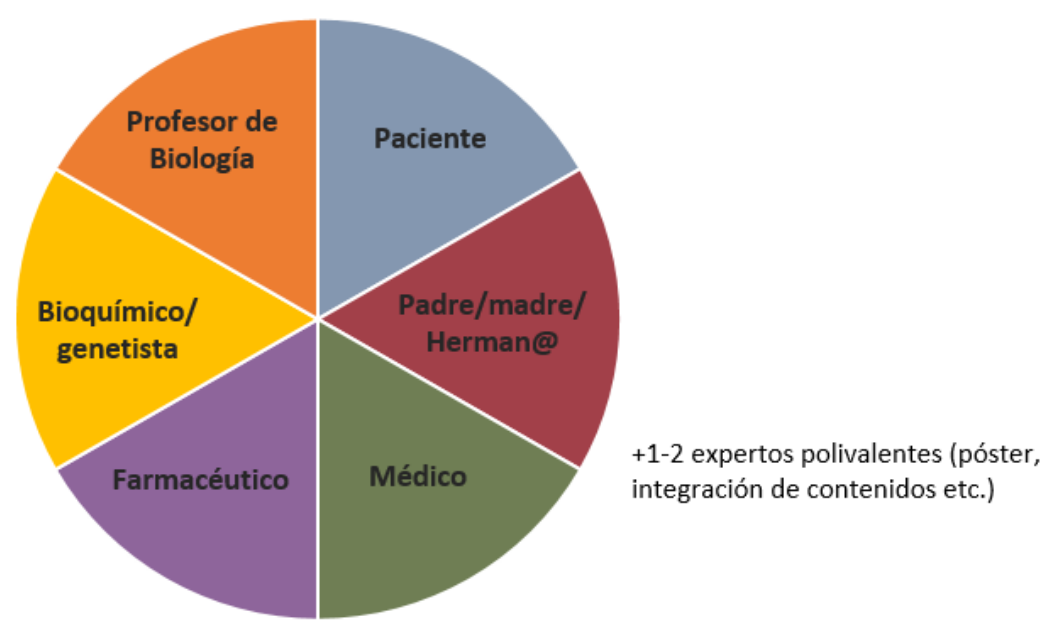

Fig. 1 Distribución de roles para la teatralización 
Teatralización en grupos tutorizados de Biología en Medicina:una estrategia diferente para el aprendizaje colaborativo

Se estableció un calendario de tutorías para la supervisión de los avances en las tareas de investigación, de asistencia obligatoria y los avances mínimos establecidos para cada sesión.

\subsection{Evaluación de la actividad}

Para la evaluación se utilizó una escala de calificación (tabla 1), que integra las cualidades de la ejecución y la escala para indicar el nivel de logro. Se evaluaron 12 criterios en una escala del 1 (mínimo) al 4 (máximo) para finalmente normalizar la nota sobre 1 punto que representa de la nota final de la asignatura. En ella se valoró conjuntamente todos los materiales proporcionados, la representación y la respuesta a las cuestiones planteadas tras la presentación.

Tabla 1. Escala de calificación

\begin{tabular}{|c|c|c|c|c|c|}
\hline & CRITERIOS EVALUADOS & 1 & 2 & 3 & 4 \\
\hline 1 & El trabajo escrito es formalmente correcto & & & & \\
\hline 2 & El trabajo escrito desarrolla todos los aspectos solicitados & & & & \\
\hline 3 & El póster está bien estructurado y transmite los aspectos clave del tema & & & & \\
\hline 4 & Los alumnos distribuyen equitativamente la carga de trabajo & & & & \\
\hline 5 & Los alumnos asisten a las reuniones con los objetivos de cada sesión alcanzados & & & & \\
\hline 6 & La terminología utilizada es apropiada y se interpreta su significado correctamente & & & & \\
\hline 7 & Se aprecia una profunda comprensión sobre el orgánulo afectado y la causa genética & & & & \\
\hline 8 & $\begin{array}{l}\text { Los alumnos demuestran comprender la diversidad funcional de los afectados y las } \\
\text { consecuencias para los familiares }\end{array}$ & & & & \\
\hline 9 & Cada rol se relaciona de manera apropiada con los otros roles representados & & & & \\
\hline 10 & $\begin{array}{l}\text { Los alumnos expresan correctamente las consecuencias éticas de los hallazgos genéticos } \\
\text { en su estudio }\end{array}$ & & & & \\
\hline 11 & $\begin{array}{l}\text { Se profundiza en las necesidades de afectados y familias y se conocen las asociaciones } \\
\text { existentes }\end{array}$ & & & & \\
\hline 12 & $\begin{array}{l}\text { El trabajo demuestra el análisis crítico de la problemática planteada y se responde } \\
\text { apropiadamente a las preguntas planteadas }\end{array}$ & & & & \\
\hline
\end{tabular}

Además, se distribuyó un cuestionario mediante "Formularios Google” vía enlace en el Aula Virtual que incluía preguntas sobre el esfuerzo requerido para la ejecución del proyecto, sobre el desarrollo del proyecto y sobre su valoración del trabajo en equipo. Las preguntas sobre estos aspectos se plantearon como elementos Likert. Se establecieron 5 niveles de respuesta, pero los enunciados en ellos eran diferentes por lo que no se aplicó la suma propia de las escalas Likert. Por último se solicitó que aportaran palabras que piensan ahora al pensar en enfermedades raras y sugerencias. 


\section{Resultados}

\subsection{Representación de los proyectos.}

Los alumnos participantes desarrollaron 5 representaciones teatrales ante uno o dos profesores de la asignatura, según horarios y uno o dos de los grupos restantes. Todos ellos firmaron, de manera voluntaria, su consentimiento para la realización de fotografías para la difusión de la actividad realizada (Fig. 2). En su tarea de investigación, dos de los grupos solicitaron realizar el trabajo sobre temas no incluidos en el listado, que tras valorarse por el docente, fueron aceptados: la ataxia de Friedrich (grupos 2) y el síndrome MELAS (grupo 5), además del síndrome de la triple A (grupo 1), el síndrome de Hunter (grupo 3) y la Neuropatía óptica de Lebber (grupo 4).

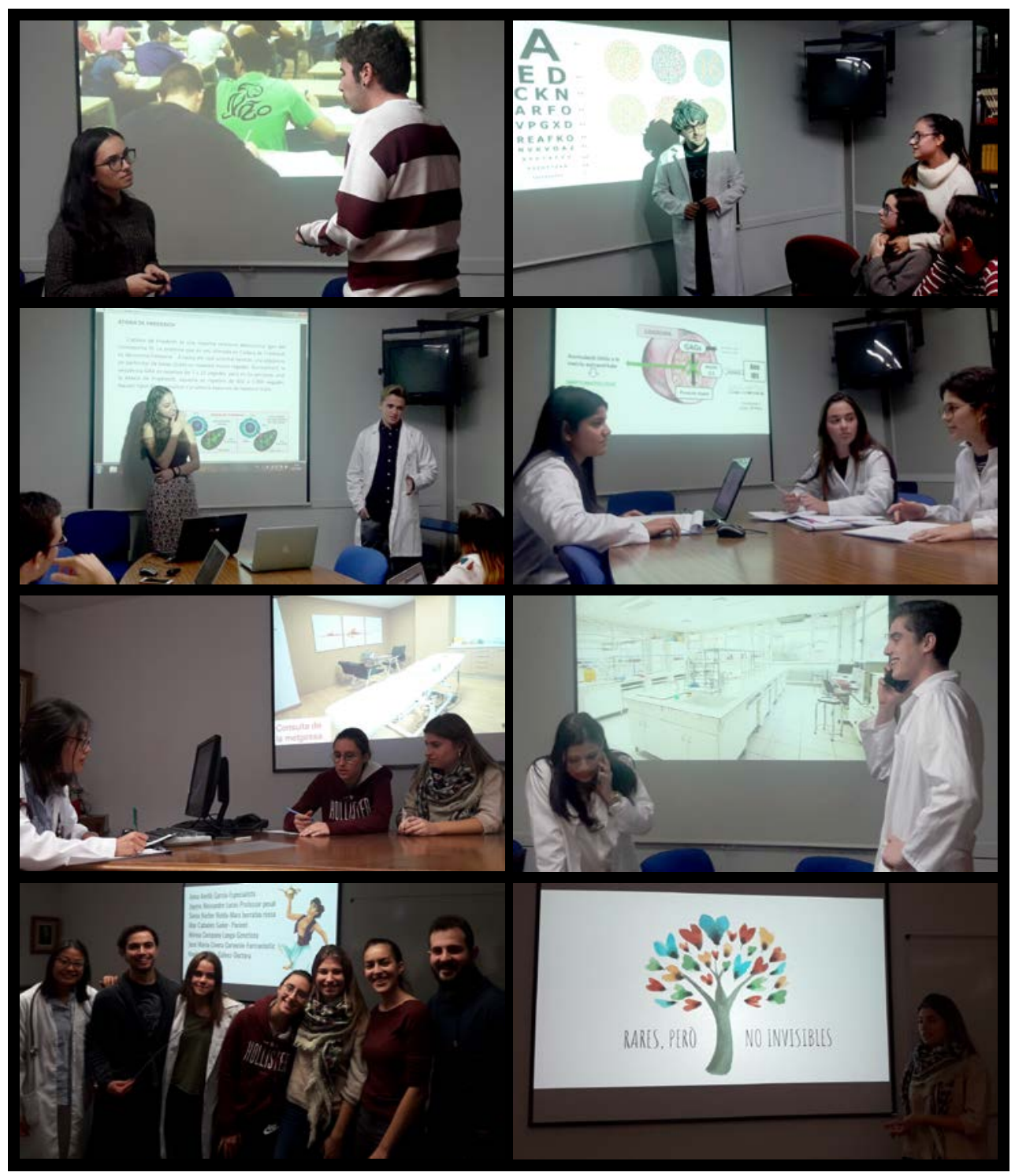

Fig. 2. Fotografías realizadas durante las representaciones teatralizadas 
El primer resultado destacable fue que, a pesar de tener la posibilidad de que algún miembro de cada equipo no tuviera que actuar, el 100\% de los alumnos decidieron adoptar un rol de actor además de trabajar en la integración de contenidos y en la preparación de los documentos físicos a presentar. Todos los grupos se adaptaron al tiempo y formato previstos.

\subsection{Consecución de los objetivos.}

Los objetivos propuestos se alcanzaron muy satisfactoriamente y los alumnos, de acuerdo a la escala de calificación, obtuvieron puntuaciones entre el 0,86 puntos y 1 punto. Las historias concebidas para transmitir los contenidos trabajados lograron incorporar en todos los casos apropiadamente:

- La fisiopatología de la enfermedad: con la interpretación de los síntomas clínicos en términos comprensibles y médicos simultáneamente, representando físicamente aquellos en que era posible y describiendo aquellos no interpretables.

- La genética de la enfermedad, con especial atención al tipo de herencia y el consiguiente estudio genético y consejo para los posibles familiares afectados

- La biología organular: la manera de incorporar los contenidos teóricos de biología celular fue sorprendente. El grupo 1 reflejó un flash-back en que los familiares protagonistas habían estudiado medicina y recordaban sus clases. El grupo 2 reflejó un matrimonio en que la médico que está trabajando con la familia afectada está casada con un profesor de biología que le recuerda los contenidos. El grupo 3 realizó un sketch en el que nos representaron a los profesores de la asginatura explicando juntos el orgánulo en cuestión. El grupo 4 unió la lección de biología al papel de genetista, aportando una explicación completa para ayudar al papel del médico. En el grupo 5, el familiar del afectado acudió a la facultad para recibir del profesor la información necesaria.

- Situación actual y perspectivas futuras: todos los estudiantes encontraron una o varias asociaciones de afectados en la Comunidad Valenciana o a nivel nacional. Varios grupos contactaron con ellas para mejorar su comprensión sobre las dificultades reales que afrontan los afectados. Los participantes encontraron tratamientos farmacológicos novedosos y/o ejemplos de nueva terapias génicas. Con estos resultados cabe decir que para el profesorado ha sido una experiencia muy grata y que las presentaciones, además de amenas, fueron muy completas y detalladas.

\subsection{Evaluación de los participantes}

De las 38 encuestas anónimas distribuidas se recibieron 21 respuestas voluntarias y anónimas. De ellas, se extrajo que, a pesar de tener que elaborar un trabajo escrito, una presentación guía, un póster de presentación en congreso y realizar una representación

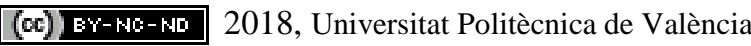

Congreso IN-RED (2018) 
teatralizada, la percepción del esfuerzo necesario para ejecutar todas las tareas en 3 semanas, fue "medio" para más de la mitad de los alumnos con un 52,4\%. Fue "bastante" para el 33,3\% y solo "mucho" para un 14,3\% de los alumnos (Fig. 3). El sistema de teatralización logra, en opinión de los estudiantes, el trabajo en equipo, en un 87,5\% de los casos. Sólo un alumno piensa que el trabajo no se ha distribuido de manera equitativa dentro de su grupo (Fig. 4).

Sobre el desarrollo del proyecto, el diseño ha sido bien valorado por los estudiantes con excepción de un alumno, y los temas tratados y el soporte recibido por el tutor también han sido muy bien valorados con el $100 \%$ de las opiniones entre de acuerdo y muy de acuerdo. El 90\% valoran positivamente la actividad desarrollada y solo un alumno no es partidario de extender este tipo de actividades a otros programas de tutelados; si bien el $14 \%$ se manifestaron “neutros”, más del 80\% está a favor de extenderlo.

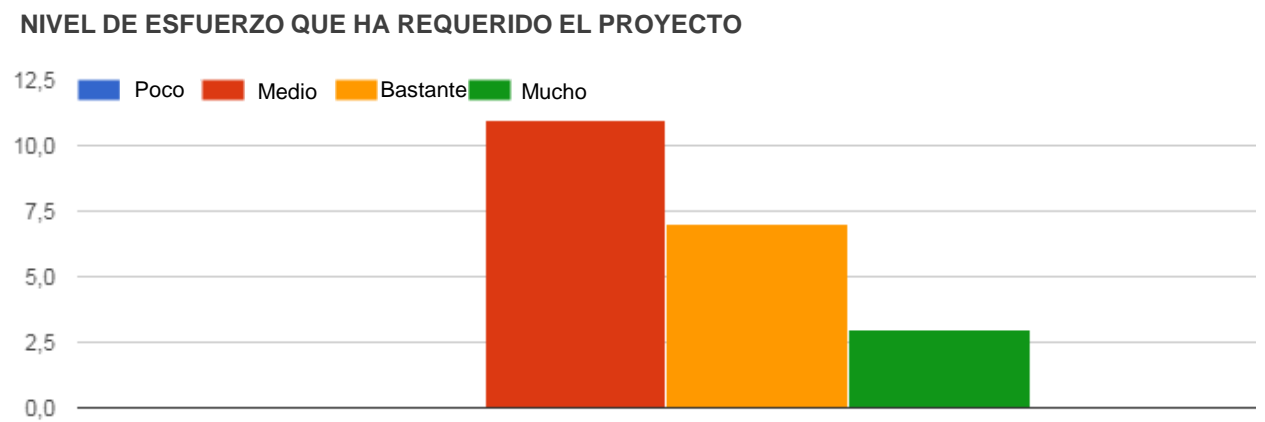

(en comparación con la tutela de otras asignaturas)

Fig. 3. Valoración del nivel de esfuerzo que ha requerido el proyecto

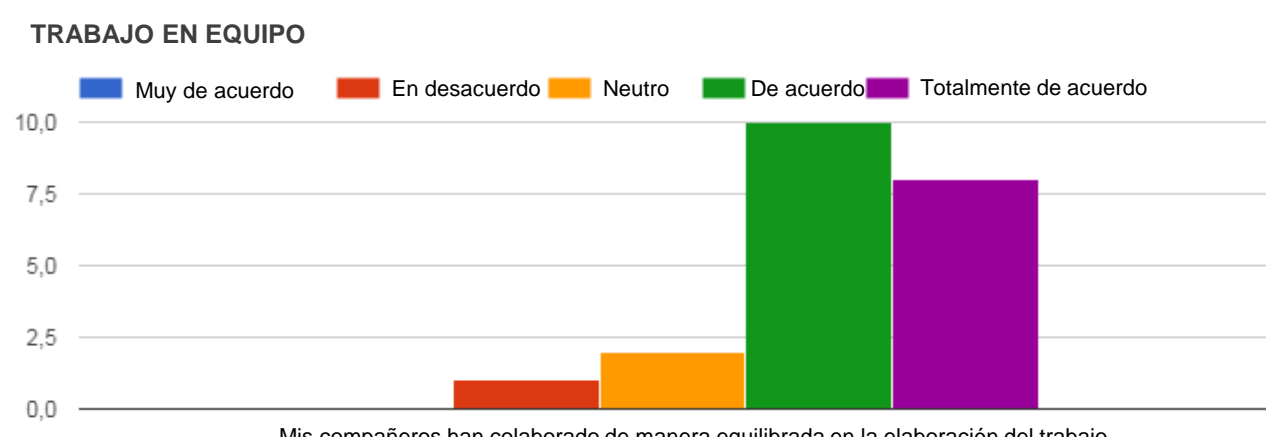

Mis compañeros han colaborado de manera equilibrada en la elaboración del trabajo

Fig. 4 Valoración del trabajo en equipo 
Teatralización en grupos tutorizados de Biología en Medicina:una estrategia diferente para el aprendizaje colaborativo

El resultado menos positivo es sobre la temporalización, ya que sólo el 76,2\% valoran positivamente su adecuación, con un $14,3 \%$ de opiniones neutras y un 9,5\% en desacuerdo con que haya sido adecuado a las necesidades (Fig. 5).

Por último, en relación a las palabras que piensan ahora al oir hablar de enfermedades raras, podemos resumir que: "investigación” o "desarrollo de investigación” e "investigación en genética” fueron nombradas 10 veces. Referencias a las dificultades, el desconocimiento, la falta de información y de inversión, y a la ausencia de cura se nombraron 12 veces. Llama la atención la cantidad de referencias a emociones: entre ellas, sorprende la relación a la frustración con expresiones como "marginales", "dolor” (x3), "pena” (x2), "incertidumbre”, "impotencia", e incluso "rabia”. Con un carácter más positivo, son numerosas las que hacen referencia a la "ayuda” (x4), "asociaciones”, "tolerancia”, "respeto” (x2) y "fortaleza” (x3). Finalmente, las expresiones sobre investigación pero con un carácter positivo motivador, se encontraron en 12 comentarios, del tipo "necesario" (x3), "importante” (x2), "curioso" (x2), “interesante” (x2), "colaborativo”, y “futuro” (x2).

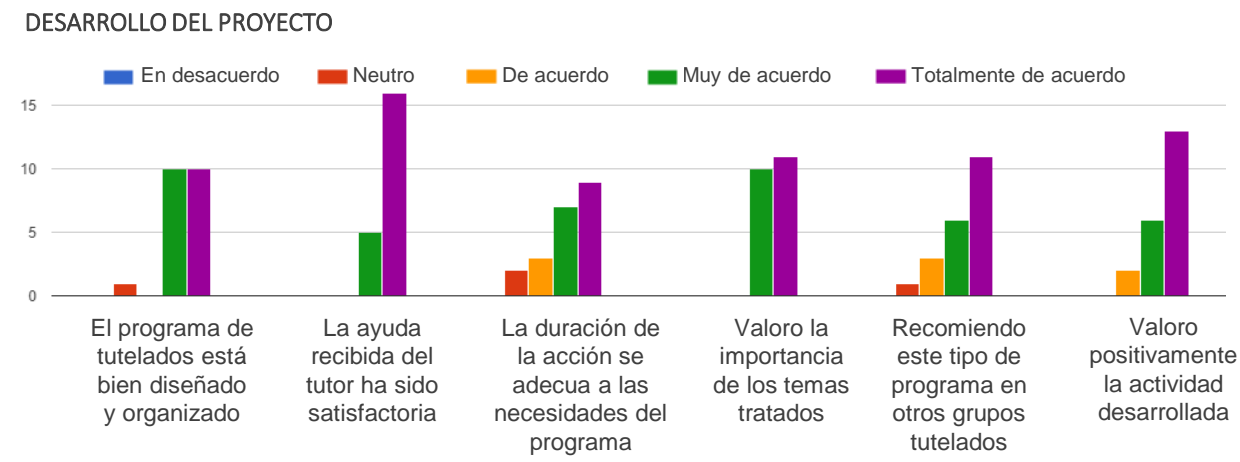

Fig. 5 Evaluación del desarrollo del proyecto.

\section{Conclusiones}

La teatralización como actividad ha tenido una acogida excelente entre los alumnos. Es muy importante que, indirectamente, ha logrado mejorar el reparto de tareas en el trabajo, por lo que valoramos muy positivamente su uso para lograr la cooperación en la creación de conocimiento para nuestros estudiantes. El reparto de roles, en que cada uno de los actores es experto en el desarrollo de su parte del trabajo, ayuda al estudiante a no sentir la carga de tener que crear un trabajo completo pues comienza con la especialización en su area concreta; sin embargo, el punto de teatralizar ayuda sin sensación de sobreesfuerzo a la integración de todos los contenidos. Los roles adjudicados tenían todos asociados tareas de investigación solapantes: no se pueden aprender los síntomas clínicos sin su explicación cotidiana, no se puede comprender el fenotipo de la enfermedad sin comprender su causa genética. Esto, a su vez, convierte la propia creación de la obra en una especie de puzle de

(cc) EY-NC-ND 2018, Universitat Politècnica de València

Congreso IN-RED (2018) 
Aaronson en que cada experto aporta y recibe, creando un flujo de conocimiento extraordinario. Por estas razones, el profesorado valora de manera conjunta la teatralización como una estrategia pedagógica muy apropiada. Es evidente que cuantas más estrategias tengamos a nuestra disposición para lograr un objetivo didáctico concreto, más probable es lograr el éxito (Pérez-Aldeguer, 2017).

Como estrategia para el próximo curso, dado que los discentes refieren falta de tiempo para abarcar todos los aspectos del proyecto, y que las valoraciones en cuanto al esfuerzo dedicado fueron muy positivas, observamos el deseo de hacer un trabajo más profundo y detallado. Así, nos planteamos modificar los requisitos de documentación a entregar para que resulte menos laborioso. De esta manera, los alumnos dispondrían de más tiempo para ahondar en los conocimientos que necesitan interiorizar.

Por otra parte, y en relación a la evaluación de competencias, en este curso hemos utilizado el sistema de "Escala de Calificación" para caracterizar los criterios de evaluación a introducir y decidir los grados de consecución. Sobre esta escala, ya trabajada, resultará más sencillo construir un rúbrica, en la que se describirán los objetivos a alcanzar en cada grado y se modificará el peso relativo de los criterios, que en este caso, fue homogéneos para todos los parámetros incluidos. La finalidad de construir una rúbrica meditada y ensayada a partir de una escala previa, es familiarizarse con el sistema de calificación; esto se ha logrado, y sobre ello, podemos construir una herramienta de evaluación que pueda ser distribuida a los alumnos antes de comenzar el proyecto, de manera que tengan claro qué y cómo se va a evaluar desde el principio de la actividad.

Es importante señalar que el hecho de tener que realizar una representación teatral, obliga al alumno a lograr un dominio de los contenidos muy importante y crea en él la necesidad de comprender los aspectos que va a tratar, para alcanzar la seguridad necesaria para teatralizar; dado que están en primer curso del grado, requiere de amplias explicaciones en las sesiones de tutoría para proporcionar una primera aproximación simplificada, a conceptos que tratarán en cursos más avanzados. Esto nos obliga a reflexionar: la innovación y traslación de la responsabilidad de aprender a los alumnos, obliga también al docente a estar a la altura de sus necesidades.

Podemos concluir que la actividad ha sido fructífera y que se ha trabajado de una manera mejorada en las competencias transversales. Sin duda el trabajo colaborativo adquiere una dimensión especial con este tipo de actividad, el aprendizaje se logra tanto en tutorías como con el trabajo en casa, virtual y cooperativo, e incluso se puede considerar gamificado, ya que aunque no incluye el concepto típico de clasificación por puntos, ludifica los procesos de enseñanza-aprendizaje convirtiéndolo en un proceso creativo muy formativo a la vez que gratificante. Aunando estrategias logramos aumentar la motivación intrínseca de nuestros estudiantes, generando en ellos la necesidad propia de crear conocimiento desde una perspectiva que deja huella: estamos seguros de que no olvidaran las emociones asociadas al acto teatral y por tanto, a las enfermedades raras que han presentado en este curso. 
Teatralización en grupos tutorizados de Biología en Medicina:una estrategia diferente para el aprendizaje colaborativo

\section{Referencias}

ÁLVAREZ-DOMÍNGUEZ, P. y MARTÍN-LÓPEZ, A. (2016). “El teatro como herramienta didáctica en la enseñanza de la Historia de la Educación Contemporánea” en. Revista Digital de Investigación en Docencia Universitaria 10 (1), 41-51.

ACASO, M. (2013). Reduvolution: hacer la revolución en la educación. Barcelona: Paidós

TORRE, S. y MORAES, M.C. (2005). Sentipensar: fundamentos y estrategias para reencantar la educación. Archidona: Aljibe.

DURÁN, D. (2014). Aprenseñar. Evidencia e implicaciones educativas de aprender enseñando. Madrid: Narcea

GONZÁLEZ PARERA, M. (2012). “El teatro como estrategia didáctica” en: Simposio Internacional de Didáctica de Español para Extranjeros. Argel (Argelia). Disponible en: https://cvc.cervantes.es/ensenanza/biblioteca_ele/publicaciones_centros/PDF/argel_2012/04_gonzalez .pdf [Consulta: 21 de marzo de 2018].

NAVARRO, M.R. (2010). "La Dramatización y la Promoción de Valores Democráticos en la Enseñanza Superior” en XXIX Seminario Interuniversitario de Teoría de la Educación, Madrid (España). Disponible en: <http://redsite.es/docu/29site/4navarro.pdf > [Consulta: 21 de marzo de 2018].

PÉREZ-ALDEGUER, S. (2017). “Las artes escénicas como metodología educativa en la educación superior” en Foro de Educación 15(22), 1-7. Disponible en: <http://dx.doi.org/10.14516/fde.587> [Consulta: 21 de marzo de 2018].

PEÑA, B. (2014). Fórmulas para la innovación en la docencia universitaria. Madrid: Visión Libros.

(cc) EY-NC-ND 2018, Universitat Politècnica de València

Congreso IN-RED (2018) 